\title{
A Case of Functional Hypoparathyroidism due to Hypomagnesaemia Leading to Hypocalcaemic Seizures
}

\author{
Neville Aquilina $^{1 *}$, Vincent Bugeja ${ }^{1}$, George Bugeja ${ }^{1}$ \\ ${ }^{1}$ Karin Grech Rehabilitation Hospital, Malta \\ *Corresponding author: Neville Aquilina: nevaqui@googlemail.com
}

\begin{abstract}
OPEN ACCESS
Citation: Aquilina N., Bugeja V., Bugeja G. (2018) A Case of Functional Hypoparathyoidism due to Hypomagnesaemia Leading to Hypocalcaemic Seizures.Open Science Journal 3(1)
\end{abstract}

Received: $31^{\text {st }}$ December 2017

Accepted: $15^{\text {th }}$ March 2018

Published: $25^{\text {th }}$ March 2018

Copyright:@ 2018 This is an open access article under the terms of the Creative Commons Attribution License, which permits unrestricted use, distribution, and reproduction in any medium, provided the original author and source are credited.

Funding: The author(s) received no specific funding for this work

Competing Interests: The author have declared that no competing interests exists.

\section{Abstract:}

This case report describes an elderly lady who was maintained on a high dose of loop diuretics following resolution of her acute pulmonary oedema, which in turn led to wasting of calcium and magnesium at the nephron level. Her hypomagnesaemia further contributed to the hypocalcaemia by rendering ineffective the normal parathyroid negative feedback physiological response. This state of low calcium increased the neuromuscular excitability leading to myoclonic epilepsy which was resistant to benzodiazepines. Such hypocalcaemic tetany in this case would only be adequately addressed once the magnesium levels are repleted, as this would allow the parathyroid mechanism to function effectively and redress the calcium level as a result.

Keywords: Seizures, hypocalcaemia, hypomagnesaemia, hypoparathyroidism

\section{Case}

An 82 year old lady originally presented to the acute general hospital with a small haemorrhagic cerebrovascular accident (stroke) complicated by flash pulmonary oedema (neurogenic) which was treated with high dose loop diuretics. She was a known case of hypertension and type 2 diabetes mellitus on aspirin, perindopril, metformin and gliclazide, naturally her antiplatelet having been withdrawn with this admission. There were no neurological sequelae to the stroke sustained, but she was left on $4 \mathrm{mg}$ bumetanide daily, despite rapid resolution of 
her flash pulmonary oedema, in view of the fact that a follow up echocardiogram showed mild left ventricular hypokinesia, to safeguard against recurrence of her pulmonary congestion.

Following transfer to our facility at Karen Grech Rehabilitation Hospital for post - acute care, she developed generalized, coarse, myoclonic jerking - involving head titubation, jaw jerking and repetitive clonic movements of all limbs occurring rather acutely a couple of weeks after her arrival. This was initially treated with rectal diazepam, to no significant effect, whilst an urgent CT (computed tomogram) brain scan came back unremarkable (showing good resolution of her original intracranial bleed). The persistent myoclonic activity was very disruptive, even during sleep. A panel of blood tests including calcium, phosphate and magnesium was taken the following day, showing very low magnesium (0.32 mmol/L), a similarly low corrected calcium $(1.75 \mathrm{mmol} / \mathrm{L})$ with a marginally elevated phosphate $(1.42 \mathrm{mmol} / \mathrm{L})$, whilst she was also hyponatraemic $(128 \mathrm{mmol} / \mathrm{L})$ and mildly hypokalaemic $(3.3 \mathrm{mmol} / \mathrm{L})$. Unfortunately we were unable to take a parathyroid hormone (PTH) level for logistical reasons, whereas 24 hour urine studies for electrolytes were not carried out in the clinical setting as they were perceived to be an academic exercise vis a' vis stabilisation of the patient's condition.

We gave intravenously $4 \mathrm{~g}$ (16 mmoles) of magnesium sulphate and 40 millimoles of calcium gluconate in $0.9 \%$ saline over 24 hours. Within 2 days her serum calcium levels rose to $2.3 \mathrm{mmol} / \mathrm{L}$, the magnesium stabilised at 0.89 $\mathrm{mmol} / \mathrm{L}$ after a transient overshoot the day before, with all other electrolytes settling as well. Clinically, her seizures resolved quite rapidly within a couple of hours of starting the 24 hour infusion, following which she remained seizure free.

\begin{tabular}{|l|l|l|l|}
\hline Electrolyte & Baseline & Day 1 (during infusion) & Day 2 (post infusion) \\
\hline Urea $(\mathrm{mmol} / \mathrm{L})$ & 7.6 & 6.9 & 6.7 \\
\hline Creatinine $(\mathrm{\mu mol} / \mathrm{L})$ & 106 & 108 & 102 \\
\hline Sodium $(\mathrm{mmol} / \mathrm{L})$ & 128 & 130 & 133 \\
\hline Potassium $(\mathrm{mmol} / \mathrm{L})$ & 3.3 & 3.6 & 3.9 \\
Calcium $(\mathrm{mmol} / \mathrm{L})$ & 1.75 & 2.15 & 2.31 \\
\hline Magnesium $(\mathrm{mmol} / \mathrm{L})$ & 0.32 & 1.49 & 0.89 \\
\hline Phosphate $(\mathrm{mmol} / \mathrm{L})$ & 1.42 & 1.34 & 1.31 \\
\hline
\end{tabular}

\section{Discussion}

Magnesium is the second most abundant intracellular cation in humans and is involved in numerous biological processes. The kidney and intestinal tract are the principal organs involved in magnesium homeostasis. Although calcium is considered to be the major regulator of parathyroid hormone (PTH) secretion, a number of studies have demonstrated that magnesium can modulate PTH secretion in a manner similar to calcium. Especially, it has been suggested that 
intracellular magnesium depletion impairs the ability of the parathyroid glands to secrete PTH resulting in a fall in the serum PTH levels, and subsequently a fall in the serum calcium concentration [7].

With their seminal 1976 publication Rude et al. (1976) studied seventeen patients suffering from this condition in an attempt to determine the pathogenesis of hypomagnesaemia-induced hypocalcaemia. They selected participants with a mean initial serum calcium concentration of 1.7-1.9 mmol/L, whilst their mean initial serum magnesium concentration was $0.3-0.4 \mathrm{mmol} / \mathrm{L}$. Serum immunoreactive parathyroid hormone (IPTH) was measured in sixteen patients in the untreated state. Despite their severe hypocalcaemia, serum IPTH was either undetectable (less than $150 \mathrm{pg} / \mathrm{ml}$ ) or normal (less than $550 \mathrm{pg} / \mathrm{ml}$ ) in all but two patients, hinting at a paradoxically impaired PTH release with failure of the PTH negative feedback loop $[2,8]$.

We postulate that this patients' long-term high dose bumetanide (4mg daily) had resulted in severe hypocalcaemia and hypomagnesaemia following salt (or sodium) wasting in the nephron, thus negating the nephron of its capacity to reclaim these cations from the urinary filtrate. Renal calcium and magnesium reabsorption both rely on the pump mediated influx of sodium from urinary filtrate with subsequent drawing in of water, which in turn carries calcium and magnesium ions with it (a process known as solvent drag) via the paracellular route in the thick ascending limb of the loop of Henle and distal convoluted tubule $[1,5]$. Conversely, in this case, the natriuretic action of bumetanide results in all these other cations being washed out in tandem with sodium within the urinary filtrate $[1,5]$.

Albeit not in possession of a formal PTH level we found ample evidence in the literature that low magnesium effectively leads to a state of functional hypoparathyroidism and this is a well recognised phenomenon. It is known that magnesium is crucial in formation of cyclic AMP (adenosine monophosphate) both in the parathyroid glands as well as in their end-organs, such that both synthesis and secretion of PTH would be impaired [7], whilst it's activity at end organs would be blunted, in the face of a low magnesium $[7,9,10,11]$. We surmise that failure of the parathyroid mechanism to reabsorb calcium from the bone, intestines and nephron (via activation of a specific calcium pump) dangerously lowered the serum calcium level which was producing seizure activity. It follows, from such a functional hypoparathyroid state, that one might expect to find either a slightly low or possibly a normal PTH level upon serum testing, since there is both an absolute as well as a relative PTH deficiency, given that the circulating PTH is unable to carry out fully its action on end organs at a PTHreceptor level, even if this hormone is present in sufficient quantities, when contending with hypomagnesaemia. Moreover this 'reduced sensitivity' of PTH at effector end organs (gut enterocytes, nephron tubule cells and osteoclasts) might actually stimulate further hormone release from the parathyroid glands in an effort to overcome this partial metabolic block and the resultant calcium wastage (or persistence of hypocalcaemia) by means of its negative feedback loop mechanism.

Treatment with intravenous calcium and magnesium allowed the calcium level to normalize, leading to rescindment of the neuromuscular hyperexcitability and resolution of the epilepsy. Phosphate level normalized (from its borderline high level) as well, indicating that the parathyroid dysfunction had resolved. The 
magnesium level initially overshot the upper limit of normal but rapidly redistributed to within the normal range within $24-48$ hours. The patient was maintained on oral magnesium supplementation (magnesium citrate tablets at $300 \mathrm{mg}$ daily) so as to avoid recurrence of this phenomenon [6].

\section{Learning Points}

1. Hypomagnesamia causes functional hypoparathyroidism.

2. Resistant hypocalcaemia merits a search for underlying hypomagnesaemia.

3. Long term loop diuretics is a leading cause of cation (including magnesium) depletion in the elderly.

4. Always exclude electrolyte disturbances with seizures, particularly if the jerking is myoclonic in nature and there is no clouding of consciousness.

5. Magnesium and calcium rapidly redistribute to the musculoskeletal system so any overshoot during intravenous administration is transient and rapidly buffered.

\section{References:}

1. Human Physiology, $2^{\text {nd }}$ revised edition 1989, Chapter 30; Function of the kidneys. Schmidt R and Thews G, Springer Verlag publishers.

2. Clin Endocrinol (Oxf). 1976 May;5(3):209-24. Functional hypoparathyroidism and parathyroid hormone end-organ resistance in human magnesium deficiency. Rude RK, Oldham SB, Singer FR.

3. Magnesium depletion and hypermagnesaemia. In Primer on Metabolic Bone disease and disorders of mineral metabolism. 4th Ed. MJ Favus, Lippincot 1999;241-45.

4. Hypomagnesaemia masquerading as hypoparathyroidism. J Assoc Physicians India. 2006 May;54:411-2. Singh R, Bhat MH, Bhansali A.

5. Quamme GA. Renal Magnesium handling, New insights in understanding old problems. Kidney Int 1997;52: 1180-95

6. Abbott LG, Rude RK. Clinical manifestation of magnesium deficiency. Mineral, Electrolyte Metab 1993;19:290-95.

7. Petts JT. Diseases of Parathyroid gland. Hyper and hypocalcemic disorders. In: Harrison's principles of Internal Medicine. Eds. Braunwald, Fauci A et al; McGraw Hill, 2001; 2220-26

8. Rude et al., 1976 as found in John P. Bilezikian, Lawrence G. Raisz, Gideon A. Rodan - 2002 Medical; Mg Depletion and Parathyroid Gland Function

9. Hypomagnesemia-induced hypocalcemia: functional hypoparathyroidism, parathyroid hormoneand vitamin D-resistant. Klin Wochenschr. 1990 Jul 5;68(13):678-84. Leicht E, Biro G, Keck E, Langer HJ.

10. Magnesium Research journal. Mechanisms of hypocalcaemia in the clinical form of severe magnesium deficit in the human. 1992 Mar;5(1):37-44. Leicht E, Biro G.

11. Hypomagnesemic hypocalcemia. Early serum calcium and late parathyroid hormone increase with magnesium therapy. Arch Intern Med. 1977 Jul;137(7):953-5. Wiegmann T, Kaye M. 